\title{
MRCP with follow-on ERCP: Correlating imaging findings with ERCP results
}

N Mcaddy', D Christodoulou', F Chinaka1, P Berry², T Wong², S Gourtsoyianni', V Goh'

'Department of Radiology, Guy's and St Thomas' NHS Trust, London/GB 2Department of Gastroenterology, Guy's and St Thomas' NHS Trust, London/GB

\section{Learning Objectives:}

- Demonstrate correlation between MRCP and ERCP in the detection of ductal calculi, narrowing/stricture and dilatation.

- Illustrate common hepatobiliary pathology seen on MRCP and ERCP

- Highlight the additional value of MRCP in the diagnosis of extra-biliary disease.

\section{Background}

MRCP is a non-invasive method of imaging the pancreaticobiliary ductal system with the additional value of demonstrating extra-biliary pathology. ERCP is considered the gold standard for evaluation of the biliary system and allows for immediate therapeutic intervention but is not without complications. A proportion of ERCPs will be unsuccessful and MRCP can have an anticipatory role.

\section{Procedure Details \& Results}

Using PACS we identified patients having MRCP with follow-on ERCP over a 12 month period at our institution. Exclusion criteria included more than 14 days between studies and ERCP failure.

53 patients met inclusion criteria; ERCP failed in 4 therefore 49 patients were reviewed $(23$ male, 26

female). Mean patient age was 62 years. Average length of time between MRCP and ERCP was 4.2 days. Presence of choledocholithiasis, ductal narrowing or dilatation was recorded. With ERCP as the reference standard, sensitivity and specificity of MRCP was calculated (table). Our data is comparable with the literature for choledocholithiasis. Subset analysis showed increasing specificity with decreasing time interval between MRCP and ERCP ( $\leq 14$ days or $\leq 3$ days between studies)

\section{Image Findings}

Choledocholithiasis: Axial T2w (1a) and 3D coronal FSE (1b) MRCP images show a filling defect consistent with an intraductal calculus (arrow) in the distal CBD correlating with ERCP findings (arrow, 1c)
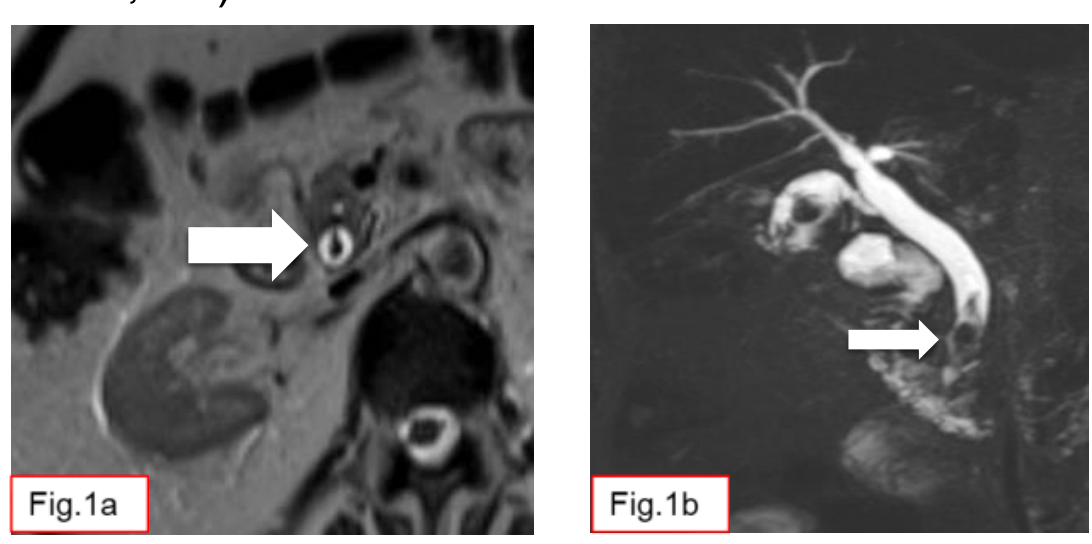

Proximal stricture: Intrahepatic biliary duct dilatation on axial T2w image (2a) with dissociation of the right and left hepatic ducts (arrow) at the hilum is highly suspicious for cholangiocarcinoma. ERCP (2b) shows intrahepatic biliary duct dilatation and 'waisting' on balloon dilatation of a segment of the stricture.

Distal stricture: $\mathrm{CBD}$ stricture (arrow) on coronal MRCP (3a) and ERCP $(3 b)$ is causing intrahepatic biliary duct dilatation. Histology from ERCP brushings confirmed malignancy.
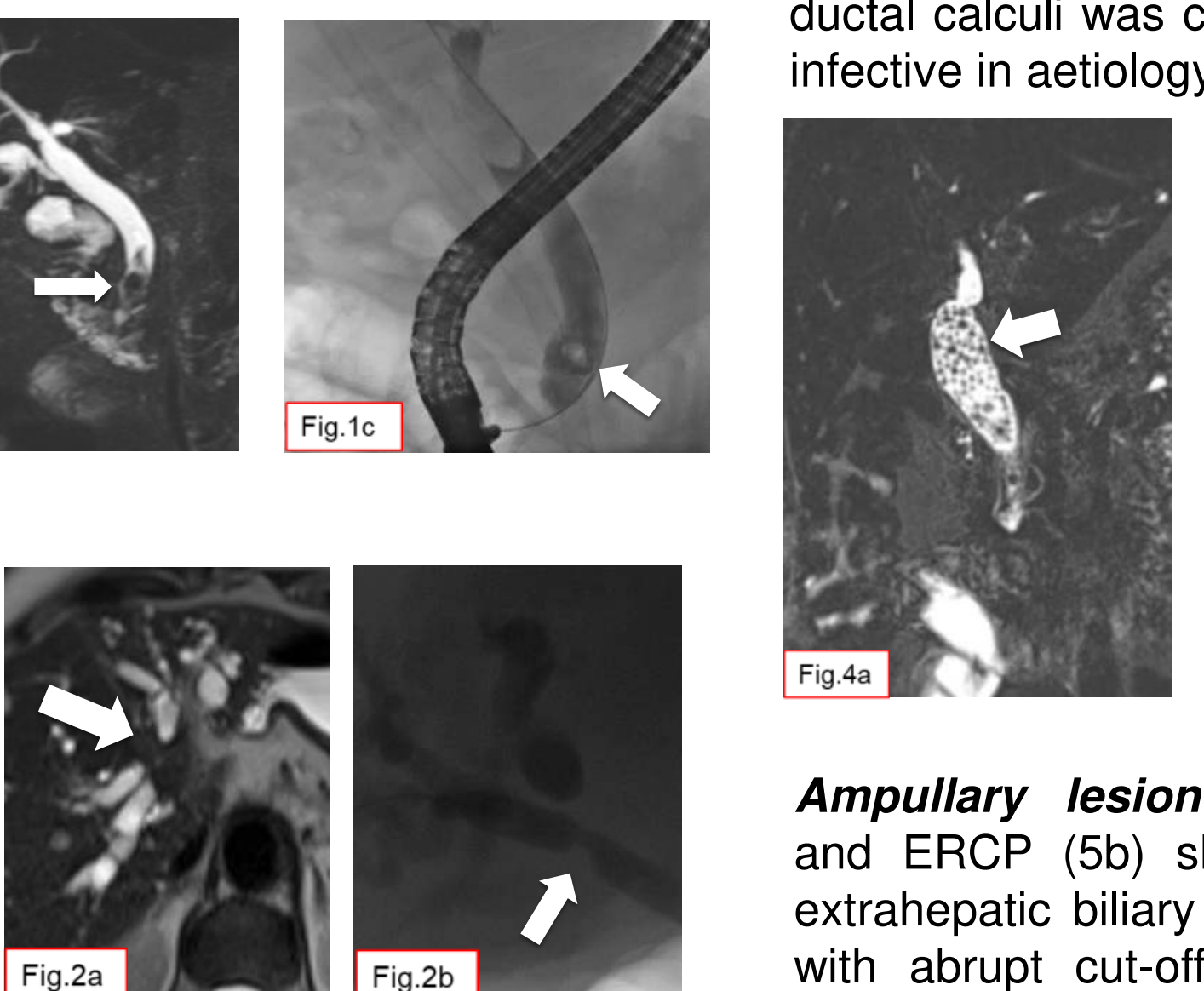

Duct Dilatation: Tiny calculi cannot be easily differentiated from multiple intraluminal air locules present in the distended CBD (4a). An air-fluid level is seen in the CBD on axial T2w image (4b, arrow). ERCP demonstrates aerobilia (4c). Absence of ductal calculi was confirmed on ERCP and aerobilia was proven infective in aetiology.
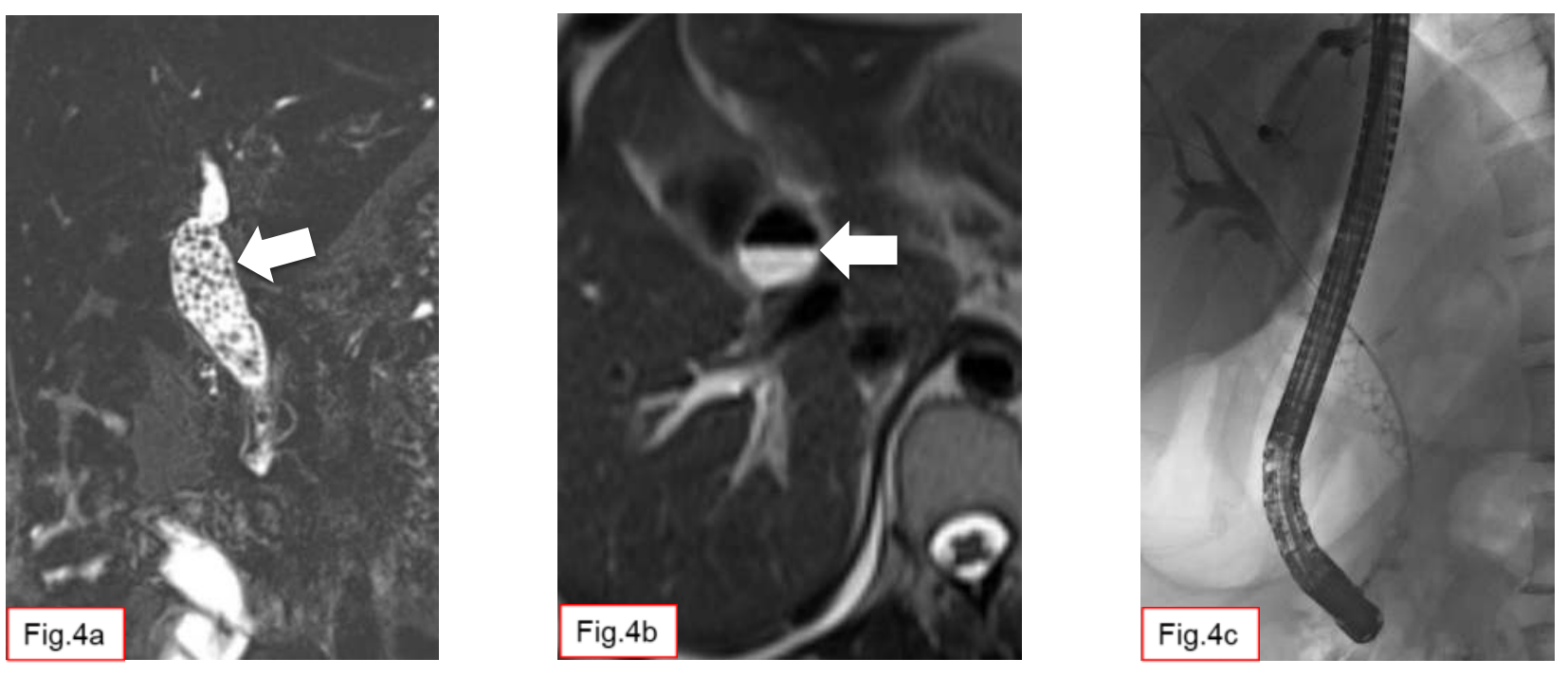

Ampullary lesion: MRCP (5a) and ERCP (5b) show intra and extrahepatic biliary duct dilatation with abrupt cut-off at the distal CBD (arrow) . A 'double duct' sign pancreatic duct dilatation. MRCP appearances are suggestive of an ampullary lesion which was visualised and biopsied

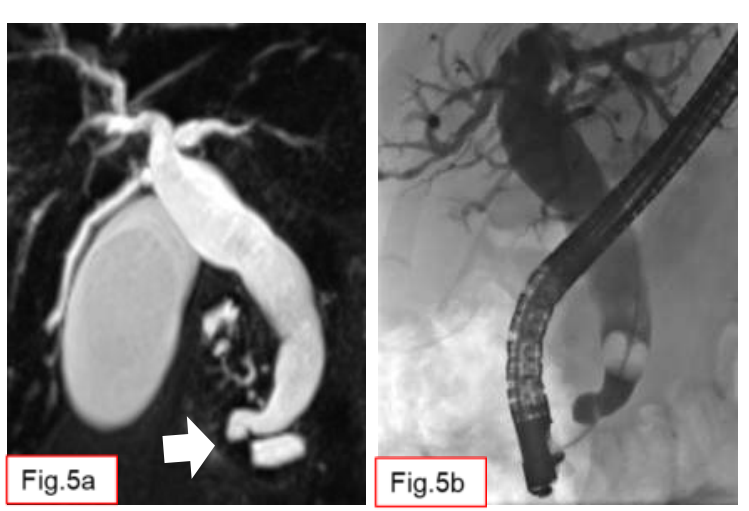
on ERCP.
A patient with metastatic CUP presented with jaundice and obstructive liver function tests. Axial T2w image (6a) shows a $13 \mathrm{~mm}$ portal lymph node $\left(^{*}\right)$ compressing the mid CBD. Liver metastases were also identified. ERCP shows smooth extrinsic compression of the mid CBD (arrow) by the lymph node. The stricture was dilated and a stent was inserted.
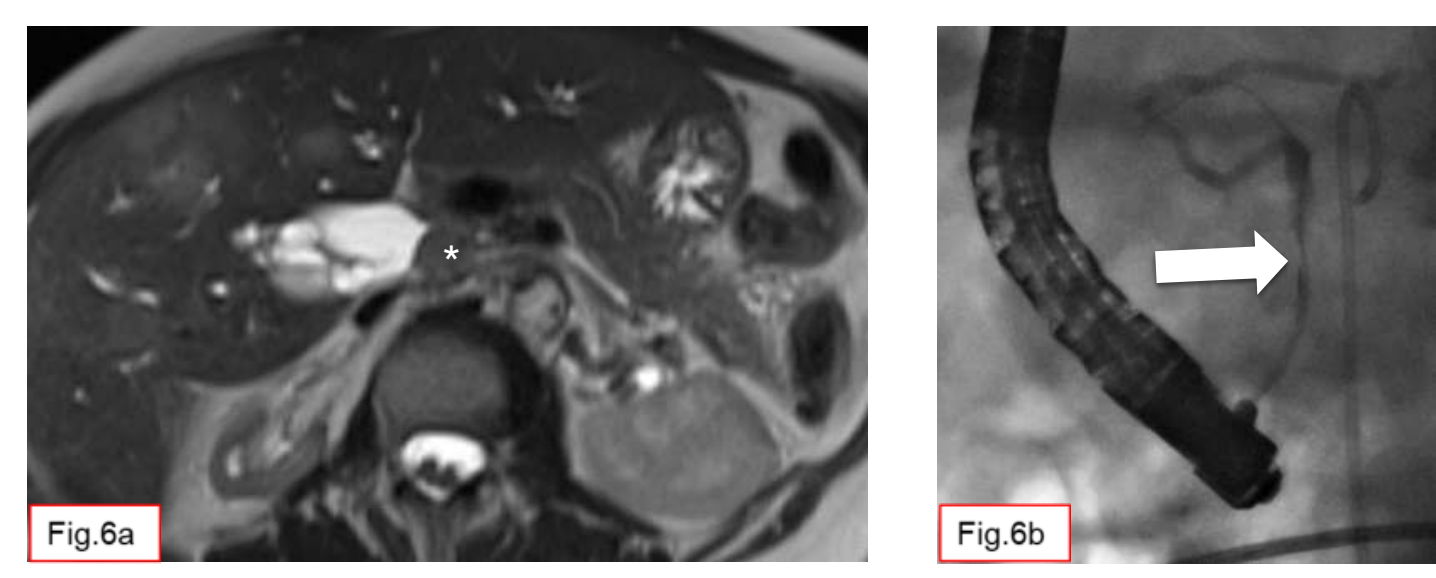

Extraductal abnormality: Axial T2w images show acalculous cholecystitis (7a) with adjacent liver abscess (7b). The biliary tree was unremarkable on ERCP (not shown).
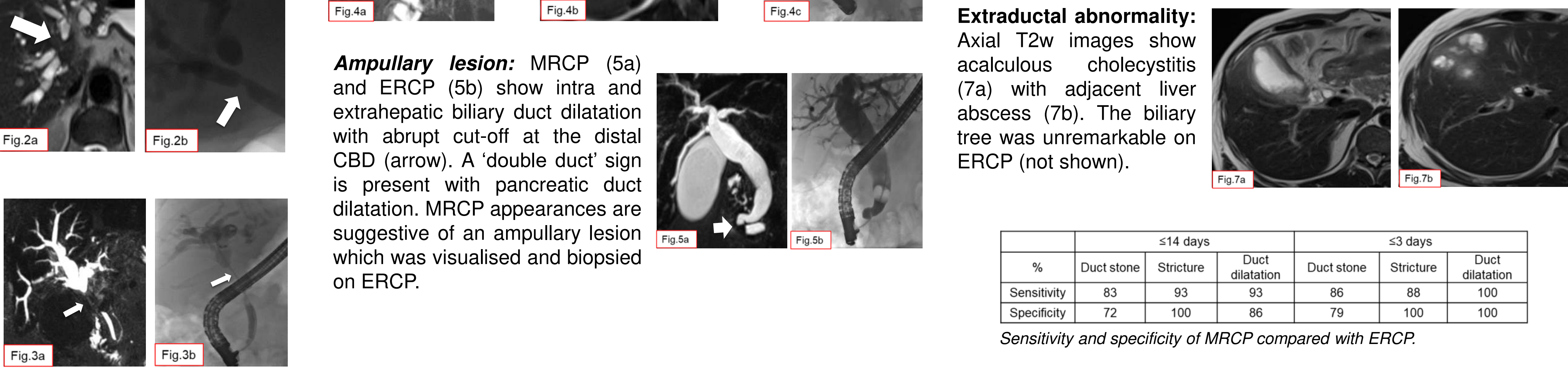

\section{Value of MRCP}

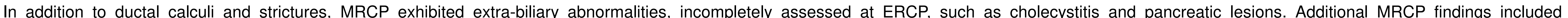

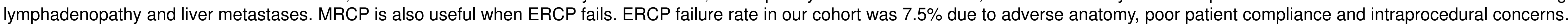

Conclusion

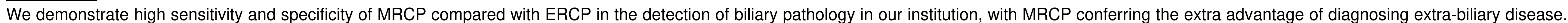
In these cases MRCP may help avoid morbidity associated with ERCP. Non-correlation between studies often reflected a more complex or evolving clinical picture. 